\title{
Does alcohol increase breast cancer risk in African-American women? Findings from a case-control study
}

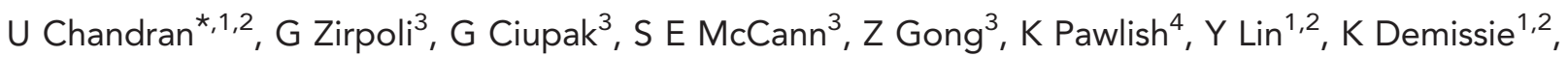 \\ C B Ambrosone ${ }^{3}$ and E V Bandera ${ }^{1,2}$
}

${ }^{1}$ Department of Medicine, Rutgers Cancer Institute of New Jersey, 195 Little Albany Street, New Brunswick, NJ 08903, USA; ${ }^{2}$ Rutgers School of Public Health, 683 Hoes Lane West, Piscataway, NJ 08854, USA; ${ }^{3}$ Department of Cancer Prevention and Control, Roswell Park Cancer Institute, Elm and Carlton Streets, Buffalo, NY 14263, USA and ${ }^{4}$ New Jersey State Cancer Registry, New Jersey Department of Health, Trenton, NJ 08625, USA

Background: Alcohol is an important risk factor for breast cancer in Caucasian women, but the evidence in African-American (AA) women is limited and results are inconclusive.

Methods: Associations between recent and lifetime drinking and breast cancer risk were evaluated in a large sample of AA women from a case-control study in New York and New Jersey. Multivariable logistic regression models provided odds ratios (ORs) and 95\% confidence intervals (Cls).

Results: There was no association between recent drinking and breast cancer risk, even when stratified by menopausal status or by hormone receptor status. A borderline decreased risk with increased lifetime consumption was found $(\mathrm{OR}=0.77$; $95 \%$ $\mathrm{Cl}$ : 0.58-1.03), which was stronger among women who drank when under 20 years of age $(\mathrm{OR}=0.65$; 95\% $\mathrm{Cl}$ : 0.47-0.89), regardless of menopausal or hormone receptor status.

Conclusion: Breast cancer risk associated with recent alcohol consumption was not apparent in AA women, while early age drinking seemed to decrease risk. This is the first investigation on recent and lifetime drinking in subgroups and drinking during different age periods in AA women. If findings are replicated, racial differences in biological pathways involving alcohol and its metabolites should be explored.

There is convincing evidence that alcohol consumption even at moderate levels increases breast cancer risk in both pre- and postmenopausal women (World Health Organization/ International Agency for Research on Cancer; IARC Monographs on the Evaluation of Carcinogenic Risks to Humans, 1998; Bandera and Kushi, 2006; World Cancer Research Fund/American Institute for Cancer Research, 2007; World Cancer Research Fund International/American Institute for Cancer Research. Continuous Update Project Report Summary, 2010; Kushi et al, 2012; Seitz et al, 2012). A recent review (Seitz et al, 2012) of 113 studies reported a modest but significant (relative risk $=1.04$; 95\% confidence interval (CI): 1.02-1.07) risk for light drinking
( $\leqslant 1$ drink per day or $\leqslant 12.5 \mathrm{~g}$ per day ethanol) compared with non-drinkers. The same review also reported breast cancer risk associated with heavy drinking ( $\geqslant 3$ drinks per day), compared with non-drinkers to be in the range of $40-50 \%$ according to the current literature. There was a positive dose-response relationship between alcohol consumption and breast cancer risk, also consistent with the World Cancer Research Fund/American Institute for Cancer Research (WCRF/AICR) expert report that showed $6 \%$ increased risk for each $10 \mathrm{~g}$ of ethanol consumed per day (World Cancer Research Fund/American Institute for Cancer Research, 2007). Hence, although alcohol should be avoided to minimise breast cancer risk, experts from both the WCRF/AICR

*Correspondence: Dr U Chandran; E-mail: chandrur@cinj.rutgers.edu

Received 3 May 2013; revised 16 July 2013; accepted 8 August 2013; published online 5 September 2013

(c) 2013 Cancer Research UK. All rights reserved 0007-0920/13 
(World Cancer Research Fund/American Institute for Cancer Research, 2007) and American Cancer Society (Kushi et al, 2012) recommend restricting alcohol intake in women to not $>1$ drink a day for breast cancer prevention, taking into account a potential protective effect for coronary heart disease.

The current evidence on alcohol and breast cancer risk is largely based on studies conducted in Caucasian populations. In a systematic review (Chandran et al, 2012) of nutritional factors and breast cancer risk in African-American (AA) women, we found only five studies (two prospective cohorts (Hiatt and Bawol, 1984; Hiatt et al, 1988) and three case-control studies (Brinton et al, 1997; Kinney et al, 2000; Zhu et al, 2003)) that presented risk estimates for alcohol and breast cancer risk stratified by race. The observed evidence in AA women was inconsistent and was limited by narrow ranges of alcohol intake and small sample sizes. More recently, a letter to the editor reported on the association between alcohol consumption, anthropometrics and breast cancer risk in a small sample of AA women (Llanos et al, 2012). A 53\% reduction in breast cancer risk associated with mostly wine consumption was reported, and a $77 \%$ reduction in breast cancer risk (for consuming 1-6 drinks compared with $<1$ drink) was reported among AA women who had low waist-to-hip ratio (Llanos et al, 2012).

To our knowledge, there are no studies on alcohol consumption and breast cancer risk among AA women that evaluate associations by menopausal or hormone receptor status, or intake at different ages. Given the limited evidence in AA women for such an important breast cancer risk factor, we investigated the relationship between recent and lifetime alcohol intakes and breast cancer risk in a large sample of AA women participating in the Women's Circle of Health Study (WCHS), a case-control study based in New York (NY) and New Jersey (NJ).

\section{MATERIALS AND METHODS}

The design and procedures followed in the WCHS have been described elsewhere (Ambrosone et al, 2009). In summary, the WCHS was a case-control study conducted in NY and NJ. Eligibility criteria for cases included AA or Caucasian females, 20-75 years of age, no previous history of cancer except for nonmelanoma skin cancer, able to understand and speak English and newly diagnosed with histologically confirmed invasive breast cancer or ductal carcinoma in situ (DCIS). Controls had the same eligibility criteria as cases except that they had no breast cancer. Study recruitment in NY ended in December 2008 while data collection in NJ pertaining to this investigation was completed in March 2012. Cases in NY were identified through hospitals with the largest referral patterns for AA women while in NJ, rapid case ascertainment methodology was employed by the NJ State Cancer Registry to identify eligible cases. African-American controls in NY were recruited through random digit dialling (RDD) and in NJ through RDD supplemented by recruitment through community sources (Bandera et al, 2013). Although WCHS included both AA and Caucasian women, because of the plethora of evidence relating alcohol and breast cancer risk in Caucasian women and the scarce literature in AA women, these analyses focus on AA women. Overall, the participation rate for those who were contacted and eligible was $78.7 \%$ and $48.2 \%$ in AA cases and controls, respectively, and $79 \%$ and $49 \%$ in Caucasian cases and controls, respectively.

Data collection. Data collection was conducted during an inperson interview, which included the administration of a main study questionnaire querying about demographics, family history, reproductive history, hormone use, lifetime alcohol consumption, physical activity and other lifestyle factors. Participants also completed a Food Frequency Questionnaire (FFQ) that queried on frequency and portion size for 125 foods and beverages including beer, red wine, white or rose wine, and liquor and mixed drinks consumed during the 12 months before diagnosis for cases and a comparable period for controls. Detailed anthropometric measurements were obtained using a standardised protocol. Data from a total of 803 AA cases and 889 AA controls were included for analyses. All participants provided informed consent, and the study was approved by the Institutional Review Boards of the University of Medicine and Dentistry of New Jersey (now Rutgers University), Mount Sinai School of Medicine and Roswell Park Cancer Institute.

Recent alcohol assessment. The FFQ provided a measure of recent alcohol intake (i.e., in the 12 months before reference date). Measuring alcohol (or ethanol) intake in grams is generally recommended as it includes both size and strength of each drink (World Cancer Research Fund/American Institute for Cancer Research, 2007) and is helpful in standardising ethanol intake across different beverages, which vary in alcohol content. Hence, alcohol intake was calculated as grams (g) of ethanol based on the assumption that there are $14 \mathrm{~g}$ of ethanol in a $12 \mathrm{oz}$ can of beer, 1 shot or $1.5 \mathrm{oz}$ of liquor or mixed drink, or $5 \mathrm{oz}$ of wine (Centers for Disease Control and Prevention, 2011). We calculated the consumption of each beverage type as a function of frequency and serving size and expressed in total g per week. The sum of consumption of all the alcoholic beverages was used to estimate total recent alcohol consumption for each participant.

Lifetime alcohol assessment. The interviewer-administered questionnaire ascertained lifetime alcohol consumption and drinking during different life periods: under 20 years, 20-29 years, 30-39 years, $40-49$ years, 50-59 years, 60 years and over. For each of these periods, women were queried about how many years they drank and amount of drinks usually consumed during that time. Based on this information, we calculated total drinks consumed during each age interval as well as total lifetime drinks by summing drinks consumed in each age interval.

Statistical analyses. Frequencies were used to compare the distribution of selected characteristics between cases and controls. Mean and median consumption levels of recent alcohol and lifetime consumption were compared between cases and controls, and $P$-values were obtained from the Wilcoxon rank sum test. Multivariable logistic regression analyses were used to compute odds ratios (ORs) and 95\% CIs. Total recent alcohol intake and consumption of individual beverages were categorised as 'non-drinker' and 'drinker' as well as ' $<14$ g per week ( $<1$ drink per week)', '14-<28g per week $(1-<2$ drinks per week)' and ' $\geqslant 28$ g per week $(2+$ drinks per week)' to evaluate associations for increasing levels of drinking. Total lifetime consumption was analysed as a binary exposure (ever/never) based on the screener question for lifetime drinking as well as 'non-drinker', 'below median drinks' and 'above median drinks' (with median $=3510$ drinks) based on computed levels in controls. There were 15 women who reported consuming alcoholic beverages at least once a week for 6 months or more, but had missing information for number of drinks consumed. Drinking during different age intervals was modelled as a binary variable (non-drinker/drinker) during each specific decade.

Multivariable models were adjusted for age, ethnicity (Hispanic or non-Hispanic), country of origin ('US born', 'Caribbean born', 'other'), education (' $<12$ th grade', 'high school graduate or equivalent', 'some college', 'college graduate', 'post-graduate degree'), age at menarche, age at menopause, menopausal status, parity, age at first birth ('0-19', '20-24', '25-30', ' $\geqslant 31$ '), breastfeeding, family history of breast cancer, history of benign breast disease, hormone replacement therapy (HRT) use, and oral contraceptive (OC) use. Models were also further adjusted for body 
mass index (BMI) and total energy intake, as well as physical activity and recent alcohol consumption (for lifetime alcohol intake). These covariates were selected for inclusion into the model based on their routine adjustment as confounders in the breast cancer literature. Nevertheless, main analyses were repeated after only adjusting for factors that differed significantly in their distribution between cases and controls. If the results remained unchanged, then estimates from the full model are reported to facilitate comparison of results across studies. BMI measures included self-reported BMI 1 year before reference date as well as measurements taken by the interviewer at the time of interview. As measured BMI and BMI based on self-reported weight and height were highly correlated $(r=0.92)$, we used the objectively measured BMI values, and substituted missing values for height and/or weight with self-reported values. Subgroup analyses included further stratification by menopausal status, oestrogen receptor (ER)/progesterone receptor (PR) status (using controls as reference in polytomous logistic regression analyses), BMI (stratified as $>25 \mathrm{~kg} \mathrm{~m}^{-2}, \quad 25-29.9 \mathrm{~kg} \mathrm{~m}^{-2}, \geqslant 30 \mathrm{~kg} \mathrm{~m}^{-2}$ ) and smoking status 1 year before reference date (stratified as never smoker, former smoker, current smoker). We were not able to conduct analyses stratified by $\mathrm{ER}+/ \mathrm{PR}-$ and $\mathrm{ER}-/ \mathrm{PR}+$ tumours as the sample sizes for these subtypes were small.

In sensitivity analyses, models were evaluated after excluding HRT users $(n=219)$, DCIS cases $(n=119)$ and community controls $(n=339)$. There were also 317 women who self-reported recent drinking on the FFQ but reported being a never drinker for the lifetime alcohol question during the interview. Similarly, at least 17 women appeared to have consumed alcohol during their reference age, but did not report drinking on the FFQ. To evaluate the impact of these discrepancies, we repeated analyses after excluding these 334 women.

All analyses were conducted using SAS version 9.2 (Cary, NC, USA).

\section{RESULTS}

There were no meaningful differences between cases and controls with regard to frequencies of education, age at menarche, parity, breastfeeding history or BMI (Table 1). A higher proportion of cases reported a family history of breast cancer, personal history of benign breast disease and HRT use. Proportions of OC use and menopausal status were very similar in cases and controls. The distributions of recent and lifetime alcohol intakes for different alcoholic beverages are presented in Table 2. Overall, reported recent alcohol consumption in this study of AA women was very low, with median intake of around $16 \mathrm{~g}$ of ethanol in a week in cases and controls who reported being alcohol consumers. Among drinkers, mean intakes of total ethanol from recent drinking and beer were higher among controls than in cases, whereas the opposite was true for ethanol consumed from wine and liquor.

Associations between recent alcohol consumption and breast cancer risk are presented in Table 3. There was no clear relationship between recent alcohol drinking and breast cancer risk, both when evaluated compared with non-drinkers $(\mathrm{OR}=0.95 ; 95 \% \mathrm{CI}: 0.77-1.17)$ or for increasing levels of consumption $(\mathrm{OR}=0.95 ; 95 \% \mathrm{CI}$ : $0.65-1.39$, comparing $>28 \mathrm{~g}$ per week $v s<14$ g per week). There was also no evidence of an association between drinking any specific alcoholic beverage and disease risk, although the risk estimate for consuming $>2$ mixed drinks or liquor a week compared with $<1$ drink was strong, albeit statistically nonsignificant ( $\mathrm{OR}=1.53$; 95\% CI: 0.84-2.79).

There was a suggestion of decreased breast cancer risk for women in the highest category of lifetime drinking compared with non-drinkers $(\mathrm{OR}=0.77 ; 95 \%$ CI: $0.58-1.03)$, although the
Table 1. Distribution of selected characteristics for breast cancer among AA women participating in WCHS, $n=1692$

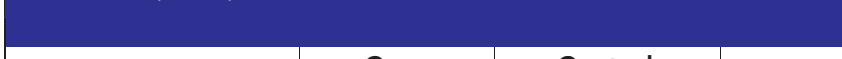

\begin{tabular}{|l|c|c|c|}
\hline & $\begin{array}{c}\text { Cases } \\
(\mathbf{n}=\mathbf{8 0 3}) \\
\mathbf{n}(\%)\end{array}$ & $\begin{array}{c}\text { Controls } \\
(\mathbf{n = 8 8 9 )} \\
\mathbf{n}(\%)\end{array}$ & $\chi^{\mathbf{2}}$ P-value \\
\hline Age at interview (years) & & & $<0.001$ \\
\hline $20-34$ & $37(4.6)$ & $71(8.0)$ & \\
$35-44$ & $162(20.2)$ & $182(20.5)$ & \\
$45-54$ & $261(32.5)$ & $320(36)$ & \\
$55-64$ & $262(32.6)$ & $272(30.6)$ & \\
$65-76$ & $81(10.1)$ & $44(4.9)$ &
\end{tabular}

65-76

Education

High school graduate

Some college

College graduate

Post-graduate degree

Country of origin

United States

Caribbean countries

Other

Ethnicity

Hispanic

Non-Hispanic

Age at menarche (years)

$<12$

12-13

$>13$

$81(10.1)$

44 (4.9)

Menopausal status

Premenopausal

Postmenopausal

Parity (live births)

0

$1-2$

3-4

$>5$

Breastfeeding

Never

Ever

Family history

No

Yes

Past benign breast

disease

No

Yes

HRT use

Never

Ever

$118(14.7)$

241 (30)

$213(26.5)$

$141(17.6)$

$90(11.2)$

112 (12.6)

227 (25.5)

259 (29.1)

$180(20.2)$

$111(12.5)$

\begin{tabular}{|c|c|c|c|}
\hline Oral contraceptive use & & & 0.38 \\
\hline $\begin{array}{l}\text { Never } \\
\text { Ever }\end{array}$ & $\begin{array}{l}333(41.5) \\
470(58.5)\end{array}$ & $\begin{array}{l}387(43.6) \\
501(56.4)\end{array}$ & \\
\hline BMI & & & 0.73 \\
\hline $\begin{array}{l}\text { Underweight/Normal } \\
\text { Overweight } \\
\text { Obese }\end{array}$ & $\begin{array}{l}151(18.8) \\
235(29.3) \\
416(51.9)\end{array}$ & $\begin{array}{l}157(17.7) \\
255(28.7) \\
477(53.7)\end{array}$ & \\
\hline
\end{tabular}

Abbreviations: $\mathrm{AA}=$ African-American; $\mathrm{BMI}=$ body mass index; $\mathrm{HRT}=$ hormone replacement therapy; $W C H S=$ Women's Circle of Health Study. 
Table 2. Comparison of mean and median intakes of recent and lifetime alcohol consumption among AA women in WCHS

\begin{tabular}{|c|c|c|c|c|c|c|c|}
\hline & \multicolumn{3}{|c|}{ Cases $(n=803)$} & \multicolumn{3}{|c|}{ Controls $(n=889)$} & \multirow[b]{2}{*}{$P$-value } \\
\hline & $\begin{array}{c}\% \\
\text { Drinkers }\end{array}$ & $\begin{array}{c}\text { Mean } \pm \text { s.d. } \\
\text { (among drinkers) }\end{array}$ & $\begin{array}{l}\text { Median (among } \\
\text { drinkers) }\end{array}$ & $\begin{array}{c}\% \\
\text { Drinkers }\end{array}$ & $\begin{array}{c}\text { Mean } \pm \text { s.d. } \\
\text { (among drinkers) }\end{array}$ & $\begin{array}{l}\text { Median (among } \\
\text { drinkers) }\end{array}$ & \\
\hline $\begin{array}{l}\text { Recent alcohol intake } \\
\text { ( } g \text { of ethanol per week) }\end{array}$ & 37.36 & $48.37 \pm 86.50$ & 16.54 & 40.2 & $54.15 \pm 116.26$ & 16.08 & 0.18 \\
\hline $\begin{array}{l}\text { Beer intake ( } g \text { of ethanol per } \\
\text { week) }\end{array}$ & 14.61 & $23.89 \pm 50.47$ & 7 & 18.36 & $40.91 \pm 107.01$ & 5.92 & 0.03 \\
\hline $\begin{array}{l}\text { Wine intake ( } g \text { of ethanol per } \\
\text { week) }\end{array}$ & 26.9 & $35.60 \pm 66.83$ & 10.79 & 28.27 & $35.15 \pm 78.23$ & 12.10 & 0.54 \\
\hline $\begin{array}{l}\text { Liquor and mixed drinks } \\
\text { (g of ethanol per week) }\end{array}$ & 20.67 & $24.26 \pm 52.28$ & 5.92 & 22.18 & $19.50 \pm 53.91$ & 5.92 & 0.55 \\
\hline $\begin{array}{l}\text { Lifetime alcohol consumption } \\
\text { (number of drinks) }\end{array}$ & 33.13 & $5955.32 \pm 10085.80$ & 2756 & 36.04 & $9966.86 \pm 25628.76$ & 3484 & 0.09 \\
\hline
\end{tabular}

Table 3. Recent alcohol consumption and breast cancer risk among AA women in WCHS

\begin{tabular}{|c|c|c|c|c|c|c|}
\hline & & & \multicolumn{2}{|c|}{ OR1 } & \multicolumn{2}{|c|}{ OR2 } \\
\hline & Cases $(n)$ & Controls (n) & Estimate & $95 \% \mathrm{Cl}$ & Estimate & $95 \% \mathrm{Cl}$ \\
\hline \multicolumn{7}{|c|}{ Total ethanol from recent alcohol intake } \\
\hline \multirow{6}{*}{$\begin{array}{l}\text { Non-drinker } \\
\text { Drinker } \\
<14 \mathrm{~g} \text { per week } \\
14-<28 \mathrm{~g} \text { per week } \\
\geqslant 28 \mathrm{~g} \text { per week } \\
P \text { for linear trend }\end{array}$} & \multirow{6}{*}{$\begin{array}{c}503 \\
300 \\
131 \\
60 \\
109\end{array}$} & \multirow{6}{*}{$\begin{array}{c}531 \\
357 \\
153 \\
70 \\
134\end{array}$} & Ref & \multirow{3}{*}{$0.79-1.20$} & Ref & \multirow{3}{*}{$0.77-1.17$} \\
\hline & & & 0.98 & & 0.95 & \\
\hline & & & Ref & & Ref & \\
\hline & & & 1.00 & \multirow{3}{*}{$\begin{array}{l}0.64-1.56 \\
0.70-1.46 \\
\end{array}$} & 0.98 & \multirow{3}{*}{$\begin{array}{l}0.63-1.53 \\
0.65-1.39 \\
\end{array}$} \\
\hline & & & 1.01 & & 0.95 & \\
\hline & & & & & & \\
\hline \multicolumn{7}{|c|}{ Ethanol from recent beer intake } \\
\hline \multirow{6}{*}{$\begin{array}{l}\text { Non-beer drinker } \\
\text { Beer drinker } \\
<14 \mathrm{~g} \text { per week } \\
14-<28 \mathrm{~g} \text { per week } \\
\geqslant 28 \mathrm{~g} \text { per week } \\
P \text { for linear trend }\end{array}$} & \multirow{6}{*}{$\begin{array}{c}684 \\
117 \\
69 \\
22 \\
26\end{array}$} & \multirow{6}{*}{$\begin{array}{c}725 \\
163 \\
92 \\
21 \\
50\end{array}$} & Ref & \multirow{3}{*}{$0.64-1.10$} & Ref & \multirow{3}{*}{$0.62-1.06$} \\
\hline & & & 0.84 & & 0.81 & \\
\hline & & & Ref & & Ref & \\
\hline & & & 1.65 & \multirow{3}{*}{$\begin{array}{l}0.78-3.51 \\
0.41-1.45 \\
\end{array}$} & 1.67 & \multirow{3}{*}{$\begin{array}{l}0.79-3.57 \\
0.41-1.49 \\
\end{array}$} \\
\hline & & & 0.77 & & 0.79 & \\
\hline & & & & & & \\
\hline \multicolumn{7}{|c|}{ Ethanol from recent wine intake } \\
\hline \multirow{6}{*}{$\begin{array}{l}\text { Non-wine drinker } \\
\text { Wine drinker } \\
<14 \mathrm{~g} \text { per week } \\
14-<28 \mathrm{~g} \text { per week } \\
\geqslant 28 \mathrm{~g} \text { per week } \\
P \text { for linear trend }\end{array}$} & \multirow{6}{*}{$\begin{array}{c}587 \\
216 \\
109 \\
50 \\
57 \\
\end{array}$} & \multirow{6}{*}{$\begin{array}{l}637 \\
251 \\
126 \\
69 \\
56\end{array}$} & Ref & \multirow{3}{*}{$0.78-1.23$} & Ref & \multirow{3}{*}{$0.75-1.19$} \\
\hline & & & 0.98 & & 0.94 & \\
\hline & & & Ref & & Ref & \\
\hline & & & 0.87 & $0.54-1.40$ & 0.85 & $0.53-1.38$ \\
\hline & & & 1.16 & $0.71-1.88$ & 1.10 & $0.67-1.80$ \\
\hline & & & & & & \\
\hline \multicolumn{7}{|c|}{ Ethanol from recent liquor/mixed drinks intake } \\
\hline \multirow{6}{*}{$\begin{array}{l}\text { Non-liquor drinker } \\
\text { Liquor drinker } \\
<14 \mathrm{~g} \text { per week } \\
14-<28 \mathrm{~g} \text { per week } \\
\geqslant 28 \mathrm{~g} \text { per week } \\
P \text { for linear trend }\end{array}$} & 637 & 691 & Ref & \multirow{3}{*}{$0.84-1.38$} & Ref & \multirow{3}{*}{$0.83-1.36$} \\
\hline & 166 & 197 & 1.08 & & 1.06 & \\
\hline & 109 & 146 & Ref & & Ref & \\
\hline & 21 & 19 & 1.54 & $0.75-3.16$ & 1.50 & $0.73-3.08$ \\
\hline & \multirow[t]{2}{*}{36} & \multirow[t]{2}{*}{32} & 1.57 & $0.88-2.81$ & 1.53 & $0.84-2.79$ \\
\hline & & & & & & \\
\hline $\begin{array}{l}\text { Abbreviations: AA = Africal } \\
\text { Circle of Health Study. OR1 } \\
\text { cancer, HRT use, OC use, } h \\
12 \text { months before reference }\end{array}$ & $\begin{array}{l}\text { dy mass index } \\
\text { nicity, country } \\
\text { disease. OR2 } \\
\text { ontrols. }\end{array}$ & $\begin{array}{l}\text { fidence interval; } \\
\text { education, age } \\
\text { djusted for tota }\end{array}$ & none repla & $\begin{array}{l}\text { apy; } O C=c \\
\text { h, breastfee } \\
\text { ohol consur }\end{array}$ & $\begin{array}{l}\text { ptive; } O R= \\
\text { nenopausal } \\
\text { asured as al }\end{array}$ & $\begin{array}{l}\text { WCHS = Won } \\
\text { ily history of } b \\
\text { umption durin }\end{array}$ \\
\hline
\end{tabular}


association for ever drinkers compared with never drinkers was null (Table 4). Inverse statistically significant associations were further observed among women who drank alcohol when they were $<20$ years of age with an OR of 0.65 (95\% CI: $0.47-0.89$ ) compared with women who were non-drinkers in that age interval. This association was significant $(P=0.01)$ even after adjusting for multiple testing (Bonferroni correction alpha $=0.01$ ). Similarly, a reduction in risk was also suggested among women who drank after age $60(\mathrm{OR}=0.42$; 95\% CI: $0.17-1.01)$; however, the sample size was small for drinkers in this age interval, and results could have occurred by chance $(P$-value $=0.05)$. There was no association between drinking during any other age interval and breast cancer risk.

When stratified by menopausal status at the time of breast cancer diagnosis/reference date (Table 5), the ORs for premenopausal $(\mathrm{OR}=1.21 ; 95 \% \mathrm{CI}: 0.90-1.62)$ and postmenopausal women $(\mathrm{OR}=0.78 ; 95 \% \mathrm{CI}$ : $0.57-1.07)$ were in the opposite direction, albeit not statistically significant. There was no evidence of an association for increasing levels of consumption in either group. However, the inverse association observed between drinking under 20 years of age and breast cancer risk was observed in both premenopausal and postmenopausal women, although the estimate was statistically significant in premenopausal women $(\mathrm{OR}=0.60$; 95\% CI: 0.38-0.94). Similarly, the inverse association with drinking under 20 years of age was also found for both $\mathrm{ER}+/ \mathrm{PR}+$ and $\mathrm{ER}-/ \mathrm{PR}$ - tumours although borderline significance was found among women with hormone receptor-positive cancers $(\mathrm{OR}=0.64 ; 95 \% \mathrm{CI}: 0.41-0.99)$.

There was no clear evidence of effect modification by BMI or smoking status for both recent and lifetime alcohol consumption, as magnitude of ORs were generally comparable across BMI and smoking categories, with most CIs including the null value (data not shown). However, the inverse association between drinking under 20 years of age and breast cancer risk was stronger among women who were overweight $(\mathrm{OR}=0.49 ; 95 \% \mathrm{CI}$ : $0.27-0.87)$ and among never smokers $(\mathrm{OR}=0.39$; 95\% CI: $0.21-0.73)$.

There was no change in the main findings on lifetime alcohol consumption (overall or drinking under 20 years of age) and breast cancer risk when multivariable models were further adjusted for recent alcohol intake or when both recent and lifetime alcohol risk estimates were also adjusted for physical activity (data not shown). Excluding HRT users, community controls or DCIS cases also did not alter the main conclusions, although the OR for recent drinking compared with non-drinkers among postmenopausal

Table 4. Association between lifetime alcohol consumption, drinking at different age intervals, and breast cancer risk among AA women in WCHS

\begin{tabular}{|c|c|c|c|c|c|c|}
\hline & & & \multicolumn{2}{|c|}{ OR1 } & \multicolumn{2}{|c|}{ OR2 } \\
\hline & Cases $(n)$ & Controls (n) & Estimate & $95 \% \mathrm{Cl}$ & Estimate & $95 \% \mathrm{Cl}$ \\
\hline \multicolumn{7}{|c|}{ Lifetime alcohol intake } \\
\hline $\begin{array}{l}\text { Never } \\
\text { Ever } \\
\text { Non-drinker } \\
\leqslant 3510 \text { drinks } \\
>3510 \text { drinks }\end{array}$ & $\begin{array}{l}537 \\
266 \\
537 \\
150 \\
107\end{array}$ & $\begin{array}{l}568 \\
320 \\
568 \\
158 \\
156\end{array}$ & $\begin{array}{c}\text { Ref } \\
0.99 \\
\text { Ref } \\
1.14 \\
0.81\end{array}$ & $\begin{array}{l}0.80-1.23 \\
0.87-1.48 \\
0.61-1.08\end{array}$ & $\begin{array}{c}\text { Ref } \\
0.96 \\
\text { Ref } \\
1.11 \\
0.77\end{array}$ & $\begin{array}{l}0.78-1.1 \\
0.85-1.45 \\
0.58-1.03\end{array}$ \\
\hline \multicolumn{7}{|c|}{ Drinking during $<20$ years } \\
\hline $\begin{array}{l}\text { No } \\
\text { Yes }\end{array}$ & $\begin{array}{r}728 \\
75\end{array}$ & $\begin{array}{l}760 \\
127\end{array}$ & $\begin{array}{c}\text { Ref } \\
0.68\end{array}$ & $0.50-0.94$ & $\begin{array}{c}\text { Ref } \\
0.65\end{array}$ & $0.47-0.8$ \\
\hline \multicolumn{7}{|c|}{ Drinking during 20-29 years } \\
\hline $\begin{array}{l}\text { No } \\
\text { Yes }\end{array}$ & $\begin{array}{l}597 \\
206\end{array}$ & $\begin{array}{l}634 \\
254\end{array}$ & $\begin{array}{c}\text { Ref } \\
0.98\end{array}$ & $0.78-1.23$ & $\begin{array}{c}\text { Ref } \\
0.94\end{array}$ & $0.75-1.1$ \\
\hline \multicolumn{7}{|c|}{ Drinking during $30-39$ years } \\
\hline $\begin{array}{l}\text { No } \\
\text { Yes }\end{array}$ & $\begin{array}{l}595 \\
197\end{array}$ & $\begin{array}{l}627 \\
245\end{array}$ & $\begin{array}{c}\text { Ref } \\
0.94\end{array}$ & $0.74-1.18$ & $\begin{array}{c}\text { Ref } \\
0.91\end{array}$ & $0.72-1.15$ \\
\hline \multicolumn{7}{|c|}{ Drinking during $40-49$ years } \\
\hline $\begin{array}{l}\text { No } \\
\text { Yes }\end{array}$ & $\begin{array}{l}559 \\
141\end{array}$ & $\begin{array}{l}582 \\
159\end{array}$ & $\begin{array}{c}\text { Ref } \\
1.02\end{array}$ & $0.78-1.33$ & $\begin{array}{c}\text { Ref } \\
0.99\end{array}$ & $0.76-1.30$ \\
\hline \multicolumn{7}{|c|}{ Drinking during $50-59$ years } \\
\hline $\begin{array}{l}\text { No } \\
\text { Yes }\end{array}$ & $\begin{array}{r}390 \\
72\end{array}$ & $\begin{array}{r}388 \\
79\end{array}$ & $\begin{array}{c}\text { Ref } \\
1.02\end{array}$ & $0.70-1.47$ & $\begin{array}{c}\text { Ref } \\
1.00\end{array}$ & $0.69-1.45$ \\
\hline \multicolumn{7}{|c|}{ Drinking during $\geqslant 60$ years } \\
\hline $\begin{array}{l}\text { No } \\
\text { Yes }\end{array}$ & $\begin{array}{r}162 \\
10\end{array}$ & $\begin{array}{r}130 \\
17\end{array}$ & $\begin{array}{c}\text { Ref } \\
0.44\end{array}$ & $0.18-1.06$ & $\begin{array}{c}\text { Ref } \\
0.42\end{array}$ & $0.17-1.0$ \\
\hline \multicolumn{7}{|c|}{ 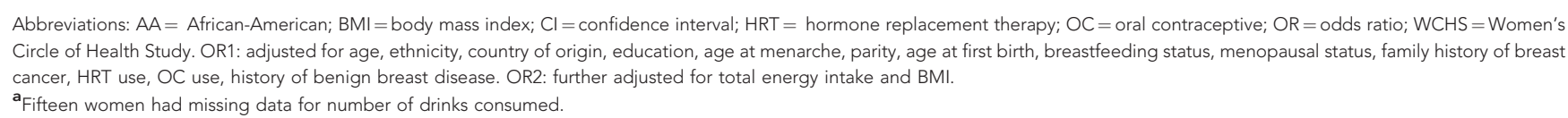 } \\
\hline
\end{tabular}


Table 5. Recent and lifetime alcohol consumption stratified by menopausal status and hormone receptor status in AA women

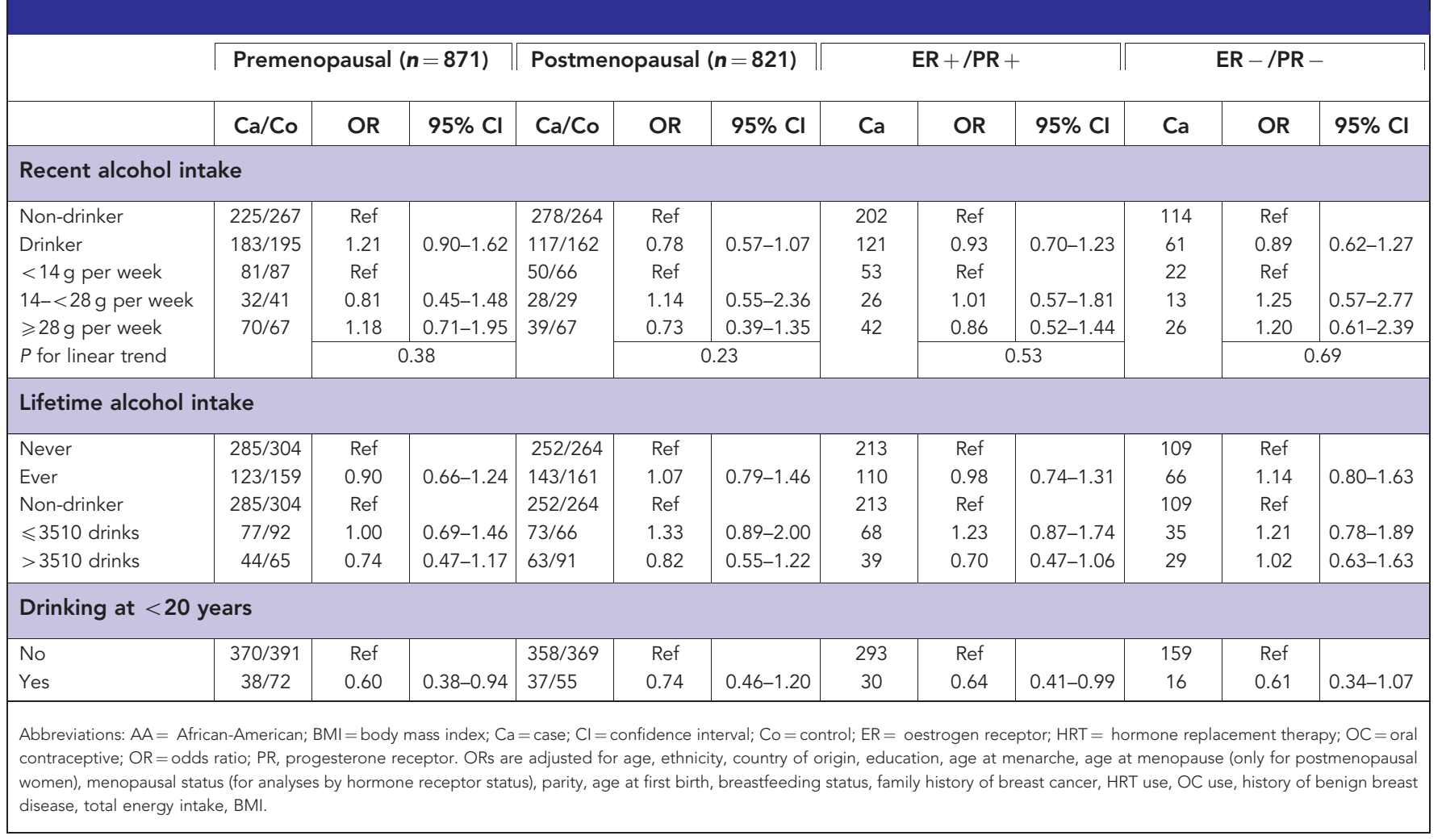

women changed from 0.78 to 0.64 when excluding community controls (data not shown). Results from the main analyses did not change in parsimonious models (data not shown).

\section{DISCUSSION}

In this case-control study involving a large sample of AA women, neither recent, light to moderate alcohol intake nor lifetime drinking significantly increased breast cancer risk, even after stratifying by menopausal or hormone receptor status. Drinking under 20 years of age was associated with a reduced disease risk when compared with non-drinkers during that age interval, which remained regardless of menopausal status and tumour receptor status. The inverse relationship observed for drinking during adolescence was also significant among overweight women and non-smokers, although magnitude of associations were generally similar across BMI and smoking levels.

Despite a larger sample size compared with previous studies, the overall null findings for recent alcohol intake and breast cancer risk were in general agreement with the current evidence in AA women (reviewed in Chandran et al, 2012). There were also no meaningful differences in risk by beverage type, except for a stronger OR observed with increasing levels of liquor consumption. In a small case-control study of AA women, an inverse association was observed between alcohol intake (mostly consisting of wine) and breast cancer risk (Llanos et al, 2012). In our study, we observed nonsignificant opposite relationships in premenopausal and postmenopausal women for drinkers compared with non-drinkers, which also could have contributed to the null findings when combining all women, although there was limited power to detect a significant association. The likelihood of spurious associations because of relatively small numbers especially among alcohol consumers cannot be discounted. As this is the first study to present risk estimates stratified by menopausal status in AA women, findings need replication.
In this study, we also found a borderline inverse association between lifetime alcohol consumption and breast cancer risk. The only other study on lifetime consumption in AA women reported null results albeit with an OR below $1(\mathrm{OR}=0.7 ; 95 \% \mathrm{CI}$ : $0.3-1.6)$ for average lifetime consumption of over $182 \mathrm{~g}$ per week (Kinney et al, 2000), but drinking in our study was much lower. It is possible that the inverse association in our study was driven by drinking during a particular lifetime period, during which time alcohol consumption may not have a detrimental effect or perhaps alcohol intake was only a marker for other factors that may reduce risk. The marginal inverse relationship observed for lifetime drinking in AA women appeared to be reflected mostly through alcohol consumption under age 20 including the adolescent period. This association remained statistically significant even when adjusted for multiple testing. Excluding HRT users also did not alter the inverse relationship. It is unlikely that this finding was affected by the participant's current age because not only was this age category applicable to all participants, but the association was found in both premenopausal and postmenopausal women. However, findings related to lifetime consumption could be spurious in view of potential recall bias described later; hence should be interpreted with caution. Some past studies mostly involving Caucasian populations have reported no risk increase for drinking at 10-15 years (Marcus et al, 2000), 15-20 years (Berstad et al, 2008), 16 years (Freudenheim et al, 1995), 18 years (Smith et al, 1994) and below 22 years (Garland et al, 1999), while other studies have shown increased risks associated with drinking below 30 years of age (Harvey et al, 1987) and during ages 18-40 years (Chen et al, 2011). Terry et al (2006) observed an inverse relationship between breast cancer risk and drinking $>15 \mathrm{~g}$ of alcohol per day (compared with non-drinkers) under age 20 $(\mathrm{OR}=0.66 ; 95 \% \mathrm{CI}: 0.41-1.06)$ and between 20 and 30 years $(\mathrm{OR}=0.57$; 95\% CI: 0.36-0.89) in women who were overweight or obese, and the sample included Caucasian women. Similarly, although the interaction between smoking and alcohol intake on breast cancer risk was not significant, perhaps the stronger inverse 
finding in non-smokers could be because of reduced exposure to toxic compounds in the absence of tobacco. However, mechanisms to explain both an increased and decreased risk of breast cancer associated with smoking have been postulated (Terry and Rohan, 2002) and, the evidence remains inconclusive. Hence, the inverse finding in non-smokers cannot be emphasised.

Racial differences in oestrogen (Taioli et al, 1996) and ethanol (Yu et al, 1995) metabolism have been noted, such that even when AA have reported heavier drinking habits, on average, they had lower levels of urinary ethanol as compared with Caucasian drinkers, which could be due to identified racial differences in distributions of genetic polymorphisms related to ethanol metabolism (Dumitrescu and Shields, 2005). A variant in the regulatory region of CYP2E1 has been associated with increased metabolic activity of this gene (McCarver et al, 1998), which could increase concentrations of acetaldehyde. The incidence of this variant has been shown to be higher in AA than in Caucasians with greater levels of metabolic activity observed mostly among obese individuals or among those who had recently consumed ethanol (McCarver et al, 1998). However, animal studies have also shown that CYP2E1 could metabolise both ethanol and acetaldehyde (Terelius et al, 1991), which could potentially result in lowered levels of acetaldehyde in the body, thus reducing toxicity. Research on the role of alcohol in promoter methylation of tumoursuppressor genes has indicated an inverse association between $p 16$ promoter methylation and lifetime drinking as well as drinking intensity (Tao et al, 2011). This inverse relationship was more prominent for intakes at earlier age periods. Hence, race-specific investigations on biological mechanisms involved in the impact of ethanol intake and oestrogen levels on breast tissue are warranted.

Certain limitations of the study should be noted. The main issue was the limited consumption of alcohol in our study because of light drinking ( $<1$ drink per day). Alcohol consumption in AA women has been generally lower than reported intakes in Caucasian women (Liu et al, 1996; US Department of Health and Human Services; Centers for Disease Control and Prevention; National Center for Health Statistics, 2012), which was also found in our study. Although the prevalence of recent alcohol consumption in our study (39\%) is similar to national estimates reporting $32 \%$ of AA women as current regular drinkers (US Department of Health and Human Services; Centers for Disease Control and Prevention; National Center for Health Statistics, 2012), only 10\% of women reported having more than half a drink per day, which is lower than levels reported among AA women in the Carolina Breast Cancer Study (Kinney et al, 2000). Therefore, we could not assess breast cancer risk associated with drinking patterns, heavy drinking ( 3 or more drinks per day) or binge drinking. There was also limited power to detect associations in stratified analyses. Low alcohol consumption in our study was not restricted to AA women. In our study, associations between recent drinking, lifetime consumption and breast cancer risk were mostly null in Caucasian women, possibly because of limited range of intake (median among drinkers was $<3$ drinks per week) even in this group (data not shown). Although majority of studies in Caucasians have shown increased breast cancer risk with increasing levels of drinking, few studies have also shown inverse or nonsignificant associations (Rosenberg et al, 1990; Friedenreich et al, 1993; Freudenheim et al, 1995).

Recall bias is also often an issue in case-control studies especially for exposures such as lifetime alcohol consumption where there could be possible errors in recalling alcohol intake from the distant past. There is some evidence showing that alcohol consumption recall (Giovannucci et al, 1993) is similar for breast cancer cases and controls. A validation study reported that recall of alcohol intake for 20 years in the past was good but not reports at 18 years of age (Dwyer et al, 1989). Another study reported that recall of alcohol intake at different age periods: 16-19 years, 20-29 years, 30-39 years, and recent consumption was very good (Longnecker et al, 1992). Specifically, a reliability study (Liu et al, 1996) using NHANES data reported that correlations for alcohol recalled from 10 years in the past for AA women were moderate and were only slightly lower than correlations observed in Caucasian women. Nevertheless, since adolescent drinking has been illegal in the United States for several years, incorrect reporting of drinking when under 20 years of age is likely. Hence, the observed inverse association between drinking under 20 years and the marginal decreased risk for overall lifetime drinking and breast cancer risk should be viewed with caution.

Mode of data collection (interviewer-administered $v s$ selfreport) could also affect reporting for certain exposures such as alcohol. In our study, $>300$ women reported to be non-drinkers on the interviewer-administered questionnaire, but disclosed alcohol intake in the self-administered FFQ, whereas only 17 women seem to have the opposite discrepancy. However, results were similar when we used the FFQ and intervieweradministered responses. Moreover, assessment of alcohol intake using self-reported methods has been shown to be both valid and reliable (Feunekes et al, 1999; Del Boca and Darkes, 2003). Nevertheless, analyses were repeated after excluding women with inconsistent data, and while there was a loss in precision as expected, study conclusions did not change.

Cases may also have changed their alcohol intake after their diagnosis and incorrectly report recent consumption. In our study, as compared with controls, greater proportion of AA cases had quit drinking since diagnosis (33.9\% vs $19.4 \%$ ), and a lesser proportion of cases had started drinking more (5.4\% vs 18\%). Drinking levels could be systematically different among controls than cases, especially with higher socioeconomic status generally observed among RDD controls. Finally, to evaluate potential selection bias arising from community control recruitment, analyses were repeated excluding them. But, main results did not substantially change. Moreover, a combination of community and RDD controls appeared to be more representative of the underlying population with regard to characteristics such as education, income, and marital status (Bandera et al, 2013). Response rates as low as $50 \%$ are not uncommon in population studies (Hartge, 2006), and case participation for studies of cancer have shown to be lower than for studies of other diseases (Olson, 2001).

Strengths of the study include the large sample of AA women allowing further evaluation by important subgroups as well as collection of both recent and lifetime drinking data. To our knowledge, this is the first and largest study of AA women to evaluate the association between both recent and lifetime alcohol intakes and breast cancer risk along with assessment of risk associated with drinking during different life periods, and stratification by several important factors.

To conclude, recent moderate alcohol intake was not associated with breast cancer risk in AA women. Lifetime drinking, particularly early age drinking, appeared to decrease risk. However, as this is the first study to report these findings in AA women, and in light of potential biases that have been discussed, no definitive conclusions can be drawn at this time. Hence, until future research is conducted to explore the role of alcohol as well as potential gene-environment interactions to evaluate racial differences in response to intake, expert guidelines of limiting alcohol consumption for cancer prevention should continue to be recommended.

\section{ACKNOWLEDGEMENTS}

This work was supported by grants from the US Army Medical Research and Material Command (DAMD-17-01-1-0334), the National Cancer Institute (R01 CA100598,P01 CA151135, 
K22 CA138563, P30CA072720, P30 CA016056), the Breast Cancer Research Foundation and a gift from the Philip L Hubbell family. The New Jersey State Cancer Registry (NJSCR) is a participant in the Centers for Disease Control and Prevention's National Program of Cancer Registries and is a National Cancer Institute Surveillance, Epidemiology, and End Results (SEER) Expansion Registry. The NJSCR is supported by the Centers for Disease Control and Prevention under cooperative agreement 1US58 DP003931-01 awarded to the New Jersey Department of Health and Senior Services. The collection of New Jersey cancer incidence data is also supported by the National Cancer Institute's SEER Program under contract N01-PC-2010-00027 and the State of New Jersey. The funding agents had no role in design, in the collection, analysis, and interpretation of data, in the writing of the manuscript, or in the decision to submit the manuscript for publication. We thank the colleagues, physicians and clinical staff in New York and New Jersey who facilitated identification and enrolment of cases into the study: Kandace Amend (i3 Drug Safety), Helena Furberg (Memorial Sloan-Kettering Cancer Center), Thomas Rohan and Joseph Sparano (Albert Einstein College of Medicine), Paul Tartter and Alison Estabrook (St. Luke's Roosevelt Hospital), James Reilly (Kings County Hospital Center), Benjamin Pace, George Raptis, and Christina Weltz (Mount Sinai School of Medicine), Maria Castaldi (Jacob Medical Center), Sheldon Feldman (New York-Presbyterian), and Margaret Kemeny (Queens Hospital Center). We also thank our research personnel at the Cancer Institute of New Jersey, Roswell Park Cancer Institute, Mount Sinai School of Medicine, UMDNJ School of Public Health, and the New Jersey State Cancer Registry, as well as our AA breast cancer advocates and community partners, and all the women who generously donated their time to participate in the study.

\section{CONFLICT OF INTEREST}

The authors declare no conflict of interest.

\section{REFERENCES}

Ambrosone CB, Ciupak GL, Bandera EV, Jandorf L, Bovbjerg DH, Zirpoli G, Pawlish K, Godbold J, Furberg H, Fatone A, Valdimarsdottir H, Yao S, Li Y, Hwang H, Davis W, Roberts M, Sucheston L, Demissie K, Amend KL, Tartter P, Reilly J, Pace BW, Rohan T, Sparano J, Raptis G, Castaldi M, Estabrook A, Feldman S, Weltz C, Kemeny M (2009) Conducting molecular epidemiological research in the age of HIPAA: a multiinstitutional case-control study of breast cancer in African-American and European-American women. J Oncol 2009: 871250.

Bandera EV, Chandran U, Zirpoli G, McCann SE, Ciupak G, Ambrosone CB (2013) Rethinking sources of representative controls for the conduct of case-control studies in minority populations. BMC Med Res Methodol 13: 71.

Bandera EV, Kushi LH (2006) Alcohol and cancer. In Nutritional Oncology, Heber D, Blackburn GL, Go VLW, Milner J (eds). 2nd edn, Chapter 11, pp 219-272. Academic Press: San Diego, CA, USA.

Berstad P, Ma H, Bernstein L, Ursin G (2008) Alcohol intake and breast cancer risk among young women. Breast Cancer Res Treat 108(1): $113-120$.

Brinton LA, Benichou J, Gammon MD, Brogan DR, Coates R, Schoenberg JB (1997) Ethnicity and variation in breast cancer incidence. Int J Cancer 73(3): 349-355.

Centers for Disease Control and Prevention (2011) Alcohol and Public Health. http://www.cdc.gov/alcohol/faqs.htm, http://www.cdc.gov/alcohol/ faqs.htm.

Chandran U, Hirshfield KM, Bandera EV (2012) The role of anthropometric and nutritional factors on breast cancer risk in African-American women. Public Health Nutr 15(4): 738-748.
Chen WY, Rosner B, Hankinson SE, Colditz GA, Willett WC (2011) Moderate alcohol consumption during adult life, drinking patterns, and breast cancer risk. JAMA 306(17): 1884-1890.

Del Boca FK, Darkes J (2003) The validity of self-reports of alcohol consumption: state of the science and challenges for research. Addiction 98(Suppl 2): 1-12.

Dumitrescu RG, Shields PG (2005) The etiology of alcohol-induced breast cancer. Alcohol 35(3): 213-225.

Dwyer JT, Gardner J, Halvorsen K, Krall EA, Cohen A, Valadian I (1989) Memory of food intake in the distant past. Am J Epidemiol 130(5): 1033-1046.

Feunekes GI, Van 't Veer P, van Staveren WA, Kok FJ (1999) Alcohol intake assessment: the sober facts. Am J Epidemiol 150(1): 105-112.

Freudenheim JL, Marshall JR, Graham S, Laughlin R, Vena JE, Swanson M, Ambrosone C, Nemoto T (1995) Lifetime alcohol consumption and risk of breast cancer. Nutr Cancer 23(1): 1-11.

Friedenreich CM, Howe GR, Miller AB, Jain MG (1993) A cohort study of alcohol consumption and risk of breast cancer. Am J Epidemiol 137(5): 512-520.

Garland M, Hunter DJ, Colditz GA, Spiegelman DL, Manson JE, Stampfer MJ, Willett WC (1999) Alcohol consumption in relation to breast cancer risk in a cohort of United States women 25-42 years of age. Cancer Epidemiol Biomarkers Prev 8(11): 1017-1021.

Giovannucci E, Stampfer MJ, Colditz GA, Manson JE, Rosner BA, Longnecker MP, Speizer FE, Willett WC (1993) Recall and selection bias in reporting past alcohol consumption among breast cancer cases. Cancer Causes Control 4(5): $441-448$.

Hartge P (2006) Participation in population studies. Epidemiology 17(3): $252-254$.

Harvey EB, Schairer C, Brinton LA, Hoover RN, Fraumeni Jr JF (1987) Alcohol consumption and breast cancer. J Natl Cancer Inst 78(4): 657-661.

Hiatt RA, Bawol RD (1984) Alcoholic beverage consumption and breast cancer incidence. Am J Epidemiol 120(5): 676-683.

Hiatt RA, Klatsky AL, Armstrong MA (1988) Alcohol consumption and the risk of breast cancer in a prepaid health plan. Cancer Res 48(8): 2284-2287.

Kinney AY, Millikan RC, Lin YH, Moorman PG, Newman B (2000) Alcohol consumption and breast cancer among black and white women in North Carolina (United States). Cancer Causes Control 11(4): 345-357.

Kushi LH, Doyle C, McCullough M, Rock CL, Demark-Wahnefried W, Bandera EV, Gapstur S, Patel AV, Andrews K, Gansler T (2012) American Cancer Society Guidelines on nutrition and physical activity for cancer prevention: reducing the risk of cancer with healthy food choices and physical activity. CA Cancer J Clin 62(1): 30-67.

Liu S, Serdula MK, Byers T, Williamson DF, Mokdad AH, Flanders WD (1996) Reliability of alcohol intake as recalled from 10 years in the past. Am J Epidemiol 143(2): 177-186.

Llanos AA, Makambi KH, Tucker CA, Shields PG, Adams-Campbell LL (2012) Alcohol, anthropometrics, and breast cancer risk in African American women. Breast J 18(4): 394-395.

Longnecker MP, Newcomb PA, Mittendorf R, Greenberg ER, Clapp RW, Bogdan G, Willett WC, MacMahon B (1992) The reliability of selfreported alcohol consumption in the remote past. Epidemiology 3(6): 535-539.

Marcus PM, Newman B, Millikan RC, Moorman PG, Baird DD, Qaqish B (2000) The associations of adolescent cigarette smoking, alcoholic beverage consumption, environmental tobacco smoke, and ionizing radiation with subsequent breast cancer risk (United States). Cancer Causes Control 11(3): 271-278.

McCarver DG, Byun R, Hines RN, Hichme M, Wegenek W (1998) A genetic polymorphism in the regulatory sequences of human CYP2E1: association with increased chlorzoxazone hydroxylation in the presence of obesity and ethanol intake. Toxicol Appl Pharmacol 152(1): 276-281.

Olson SH (2001) Reported participation in case-control studies: changes over time. Am J Epidemiol 154(6): 574-581.

Rosenberg L, Palmer JR, Miller DR, Clarke EA, Shapiro S (1990) A casecontrol study of alcoholic beverage consumption and breast cancer. Am J Epidemiol 131(1): 6-14.

Seitz HK, Pelucchi C, Bagnardi V, La Vecchia C (2012) Epidemiology and pathophysiology of alcohol and breast cancer: update 2012. Alcohol Alcohol 47(3): 204-212. 
Smith SJ, Deacon JM, Chilvers CE (1994) Alcohol, smoking, passive smoking and caffeine in relation to breast cancer risk in young women. UK National Case-Control Study Group. Br J Cancer 70(1): 112-119.

Taioli E, Garte SJ, Trachman J, Garbers S, Sepkovic DW, Osborne MP, Mehl S, Bradlow HL (1996) Ethnic differences in estrogen metabolism in healthy women. J Natl Cancer Inst 88(9): 617.

Tao MH, Marian C, Shields PG, Nie J, McCann SE, Millen A, Ambrosone C, Hutson A, Edge SB, Krishnan SS, Xie B, Winston J, Vito D, Russell M, Nochajski TH, Trevisan M, Freudenheim JL (2011) Alcohol consumption in relation to aberrant DNA methylation in breast tumors. Alcohol 45(7): 689-699.

Terelius Y, Norsten-Hoog C, Cronholm T, Ingelman-Sundberg M (1991) Acetaldehyde as a substrate for ethanol-inducible cytochrome P450 (CYP2E1). Biochem Biophys Res Commun 179(1): 689-694.

Terry MB, Zhang FF, Kabat G, Britton JA, Teitelbaum SL, Neugut AI, Gammon MD (2006) Lifetime alcohol intake and breast cancer risk. Ann Epidemiol 16(3): 230-240.

Terry PD, Rohan TE (2002) Cigarette smoking and the risk of breast cancer in women: a review of the literature. Cancer Epidemiol Biomarkers Prev 11(10 Pt 1): 953-971.

US Department of Health and Human Services; Centers for Disease Control and Prevention; National Center for Health Statistics (2012) Summary health statistics for US adults: National Health Interview Survey, 2010. Department of Health and Human Services: Hyattsville, MD, USA.
World Cancer Research Fund International/American Institute for Cancer Research (2010) Continuous Update Project Report Summary. Food, Nutrition, Physical Activity, and the Prevention of Breast Cancer.

World Cancer Research Fund/American Institute for Cancer Research (2007) Food, Nutrition, Physical Activity, and the Prevention of Cancer: A Global Perspective. American Institute for Cancer Research: Washington, DC, USA.

World Health Organization/International Agency for Research on Cancer (1998) IARC Monographs on the Evaluation of Carcinogenic Risks to Humans. Alcohol drinking.

Yu MC, Tang BK, Ross RK (1995) A urinary marker of alcohol intake. Cancer Epidemiol Biomarkers Prev 4(8): 849-855.

Zhu K, Davidson NE, Hunter S, Yang X, Payne-Wilks K, Roland CL, Phillips D, Bentley C, Dai M, Williams SM (2003) Methyl-group dietary intake and risk of breast cancer among African-American women: a case-control study by methylation status of the estrogen receptor alpha genes. Cancer Causes Control 14(9): 827-836.

This work is published under the standard license to publish agreement. After 12 months the work will become freely available and the license terms will switch to a Creative Commons AttributionNonCommercial-Share Alike 3.0 Unported License. 